\title{
Tecnura
}

\section{Alimentos funcionales: avances de aplicación en agroindustria}

\author{
Functional foods: advances of application in agroindustry \\ Daniela Arias Lamos' ${ }^{1}$ Laura Natalia Montaño Díaz², \\ María Alejandra Velasco Sánchez³, Jader Martínez Girón ${ }^{4}$
}

Fecha de recepción: 23 de noviembre de 2017

Fecha de aceptación: 23 de marzo de 2018

Cómo citar: Arias L., D., Montaño D., L.N., Velasco S., M.A. y Martínez G., J. (2018). Alimentos funcionales: avances de aplicación en agroindustria. Revista Tecnura, 22(57), 55-68. DOI: https://doi.org/10.14483/22487638.12178

\section{Resumen}

Contexto: Se realizó un estudio acerca de los compuestos bioactivos de ciertos alimentos, teniendo en cuenta los beneficios que generan a la salud humana.

Método: En este trabajo se presenta una revisión bibliográfica de las diferentes aplicaciones de los alimentos funcionales y su aplicación en la agroindustria.

Resultados: Los malos hábitos alimenticios pueden generar un déficit en el requerimiento de compuestos bioactivos importantes para el buen funcionamiento del organismo; a su vez, la demanda y la tendencia de consumo de alimentos funcionales se hace cada vez más grande debido a los múltiples beneficios y usos potenciales que se pueden obtener de éstos, junto con las necesidades nutricionales básicas que se requieren.

Conclusiones: Se evidencia el interés por realizar nuevos estudios en la obtención de este tipo de alimentos. Para esto, se deberán incorporar nuevos ingredientes en matrices alimentarias, con el fin de preservar los compuestos bioactivos y que ejecuten su principal función: que aparte de nutrir, reduzcan o controlen diversas enfermedades que en algunos casos pueden ser crónicas.

Palabras clave: compuestos bioactivos, prebióticos, probióticos, salud humana.

\section{Abstract}

Context: A study was made about the bioactive compounds of certain foods, while taking into account the benefits they generate to human health.

Method: This article presents a bibliographic review of the different applications of functional foods and their application in agroindustry.

Results: It was found that bad eating habits can generate a deficit in the requirement of bioactive compounds important for the proper functioning of the organism. On the other hand, the demand and the trend of consumption of functional foods is getting bigger due to the multiple benefits and potential uses that can be obtained from them, along with the basic nutritional needs they cover.

Conclusions: There is evidence of interest in conducting new studies about obtaining this type of food. For this, new ingredients should be incorporated in food matrices, in order to preserve the bioactive

1 Ingeniera agroindustrial. Universidad Nacional de Colombia sede Palmira, Valle del Cauca, Colombia. Contacto: daariasla@unal.edu.co

2 Ingeniera agroindustrial. Universidad Nacional de Colombia sede Palmira, Valle del Cauca, Colombia. Contacto: Inmontanod@unal.edu.co

3 Ingeniera Agroindustrial. Universidad Nacional de Colombia sede Palmira, Valle del Cauca, Colombia. Contacto: mavelascos@unal.edu.co

4 Químico, tecnólogo en alimentos, magíster en Educación, magíster en Ingeniería Agroindustrial. Profesor de la Universidad del Valle sede Palmira y docente ocasional de la Universidad Nacional de Colombia sede Palmira, Valle del Cauca. Colombia.

Contacto: jader.martinez@correounivalle.edu.co 
compounds and that they perform their main function (apart from nourish): to reduce or control various diseases that may be chronic in some cases.
Keywords: Bioactive compounds, Prebiotics, Probiotics, Human health.

\section{INTRODUCCIÓN}

Los malos hábitos alimenticios son una consecuencia de malas prácticas de alimentación y con ello el consumo deficiente de productos que aporten a la nutrición (Salas, Salcedo y Aguilera, 2013). Dietas con bajos contenidos de nutrientes, consumo elevado de alimentos y productos procesados, así como también las famosas comidas chatarras, crean efectos perjudiciales sobre la salud humana (Illanes, 2015). Existe una gran variedad de investigaciones relacionadas con los efectos de la dieta $y$ el consumo de nutrientes en el ser humano, $y$ cómo esto se relaciona con enfermedades crónicas, debido principalmente a la falta de consumo de alimentos funcionales (Chasquibol et al., 2014). Sin embargo, alrededor de la primera mitad del siglo XX, cerca de los años 1950, la buena alimentación consistió en consumir nutrientes esenciales (nutrición adecuada), mientras que años después, a finales del siglo XX, además de la alimentación adecuada, se empezó a orientar progresivamente hacia los compuestos bioactivos de los alimentos y su papel en la promoción de la salud (Trescastro y Bernabeu, 2015).

Cuando se habla de un alimento funcional quiere decir que este aporta los nutrientes básicos y que cuenta con uno o más componentes diferenciados que mejoran las funciones fisiológicas del organismo que lo consuma (Barazarte et al., 2015). El desarrollo de estos alimentos se da gracias a la incorporación de estos elementos específicos o compuestos bioactivos, como prebióticos, probióticos, péptidos bioactivos, carotenoides, vitaminas, compuestos fenólicos, fitoestrógenos, ácidos grasos o lípidos estructurados en sistemas alimentarios (Boer, Urlings y Bast, 2016; Vieira,
Barreira y Oliveira, 2016;). Estos se pueden añadir de manera natural, modificarse o mejorarse, con el fin de propiciar beneficios en la salud en $\mathrm{cu}-$ anto a desarrollo; crecimiento temprano; defensa contra el estrés oxidativo; regulación de procesos metabólicos; fisiología cardiovascular y gastrointestinal; rendimiento mental, cognitivo, físico y deportivo (Tur y Bibiloni, 2015). La producción de los alimentos funcionales va cada día en aumento a tasas del $48 \%$ anual y con valores para el mercado mundial de alrededor de $\$ 167.000$ millones (Fuentes, Acevedo y Gelvez, 2015; Eggersdorfer y Wiss, 2018).

Esta revisión presenta algunos estudios que se han reportado sobre los compuestos bioactivos presentes en alimentos funcionales y su posible aplicación y uso, así como los beneficios que puede obtener el ser humano al consumirlos.

\section{TIPOS DE ALIMENTOS FUNCIONALES}

\section{Prebióticos, probióticos y simbióticos}

Es importante incluir en la dieta diaria alimentos ricos en prebióticos y probióticos gracias a su utilidad en la disminución del riesgo y tratamiento de diversas enfermedades gastrointestinales (Olveira y González, 2016). La combinación de los probióticos y prebióticos en productos alimenticios como ingredientes funcionales se hace cada vez más interesante para la industria alimentaria (Cadena et al., 2014). Los prebióticos son ingredientes no digestivos que forman parte de los alimentos y que benefician a la persona que lo consume, mediante la estimulación del crecimiento y de la actividad de las bacterias en el colon (Azevedo et al., 2016); regulan el tránsito intestinal a la vez que favorecen 
el equilibrio de la flora intestinal (Rodríguez, Giraldo y Zuluaga, 2015), y son utilizados para prevenir enfermedades como las diarreas por antibióticos, enfermedad inflamatoria intestinal, infección por Helicobacter, encefalopatía hepática, entre otras (Olveira y González, 2016).

Potenciar la asociación de estos dos alimentos funcionales (prebióticos y probióticos) originan un efecto simbiótico que cumple ambas funciones (Reis et al., 2016). El consumo de estos alimentos eleva el promedio de vida, así pues, la flora intestinal participa en la mejora y desarrollo del sistema inmune en la niñez; también, reduce problemáticas asociadas con el cáncer de colon en la población joven por malos hábitos alimenticios, que en muchas ocasiones se llega a un estado de estrés para el sistema digestivo (Cigarran, González y Cases, 2017; Hevia, Delgado, Sánchez y Margolles, 2015; Narbona et al., 2014).

Existe evidencia sobre el efecto benéfico del yogur para la salud del huésped, pues se comporta como probiótico (Vieira, Barreira y Oliveira, 2016). Se ha investigado la viabilidad de probióticos durante la aplicación de encapsulación por liofilización para la estabilidad de cepas probióticas incorporadas en mezclas con agentes prebióticos, durante el almacenamiento de alimentos; estas técnicas protegen a los microorganismos de agentes medioambientales durante procesamiento, consumo y paso por el tracto gastrointestinal gracias a las propiedades funcionales del material de pared (Rodríguez, Giraldo y Montes, 2016; Salazar, Cortés y Montoya, 2015). Se ha estudiado la incorporación de cepas de Lactobacillus casei por medio de técnicas de impregnación al vacío en la estructura orgánica de la mora, y conservadas posteriormente por liofilización. Como resultado en la mezcla se evidenció una alta mejora en la viabilidad del microorganismo bajo este efecto, obteniendo así un alimento con cualidades simbióticas que le aportan beneficios a la salud (Rodríguez, Giraldo y Zuluaga, 2015). La ganadería como sector de la agroindustria ha centrado su interés en adicionar a las dietas del ganado productos simbióticos (mezcla de levaduras, bacterias, oligosacáridos y monensina), con el fin de evaluar características de calidad de la canal, de la carne y la utilidad que estas generan (Ribeiro et al., 2015).

\section{Alimentos enriquecidos con fibra}

El mercado de los alimentos enriquecidos con fibra se hace cada vez más grande, gracias a las funciones que este componente ofrece en nuestro organismo. Cabe resaltar que las fibras vegetales están compuestas principalmente por celulosa, hemicelulosa, lignina, pectinas y ceras (Suarez et al., 2017), de esta manera, la fuente y el tipo de fibra puede influir en las propiedades funcionales (Rana, Gupta, Rana y Bhushan, 2015), la fibra insoluble se relaciona con reducción del estreñimiento y buen funcionamiento del colon, y la fibra soluble ayuda a la reducción del colesterol y niveles de glucosa en la sangre. La industria agroalimentaria, por su parte, ha utilizado las características fisicoquímicas de las fibras para mejorar parámetros de calidad en los productos, como: características sensoriales, textura, viscosidad y vida útil, entre otros (Blanco, León y Ribotta, 2018; Yan et al., 2017).

Se evidencia la incorporación de harina de lenteja con alto contenido de fibra soluble como extensor cárnico en salchichas (Torres, González, Acevedo y Jaimes, 2016). Se han realizado aplicaciones de adición de fibras vegetales como salvado de trigo; harinas integrales multigranos, compuestas de copos de avena; grano de centeno; semillas de girasol; sésamo; linaza (Benítez et al., 2018); harina tamizada de cebada y almidón resistente para elaboración de pan. Esta última le otorga buenos resultados en la calidad tecnológica del producto de panificación, también genera cambios en la etapa de formación de la mezcla o masa y características del producto en general, como consistencia, textura, comportamiento reológico y características sensoriales (Djurle, Andersson y Andersson, 2018). El estudio realizado por 
Rana et al. (2015) para la fibra liofilizada contenida en pulpa de manzana muestra que retiene las propiedades funcionales, posee mayor capacidad de absorción de glucosa, presencia de compuestos fenólicos con acción antioxidante, de esta forma considera que la fibra dietética de frutas y en especial de la pulpa de manzana es un ingrediente funcional para productos alimenticios dietéticos.

Se ha evidenciado también, la utilización de epicarpio de frutos generados de residuos agroindustriales como fuente de fibra dietética. Okiyama, Navarro y Rodriguez (2017) expresaron los beneficios que contiene el epicarpio de cacao, el cual permite reducir y controlar niveles altos de azúcar en la sangre y de colesterol, además de mejorar los factores de riesgo de trastornos cardiometabólicos. Se sugiere que la fibra de cacao se emplee en preparaciones de galletas, pasteles o suplementos dietéticos de chocolate gracias a las propiedades similares y funcionales que tiene con la fibra de algarroba. Además, existen investigaciones sobre la incorporación de fibra dietaria obtenida de residuos de la industria de jugo de naranja (Citrus paradisi x Citrus reticulata) y fibra de manzana de anacardo (Anacardium occidentale L.) en la sustitución parcial de grasa en matrices de alimentos cárnicos (hamburguesas de pollo), en elaboración de helado y evaluación de propiedades funcionales y tecnológicas en pasta fresca (Alarcón, López y Restrepo, 2015; Crizel, Ríos, Thys y Hickmann, 2015; Guedes et al., 2016). También se ha evaluado el uso potencial de epicarpio generado como subproducto en el procesamiento del mango (Mangifera indica) en la estimación de rendimientos, obtención de alimentos concentrados, contenidos en compuestos fenólicos totales y componentes principales en el alimento (Serna y Torres, 2015).

\section{Ácidos grasos}

Dentro de los ácidos grasos que contiene la dieta del ser humano se encuentran dos grupos, unos de ellos son esenciales para el organismo y otros no. El ser humano tiene la dificultad de poder producir autónomamente los esenciales, por ende se suministran por medio de los alimentos siendo una buena fuente de energía, cuando hay deficiencia de este tipo de ácidos como el $\alpha$-linolénico (omega 3) y el linoleico (omega 6) (Lemahieu et al., 2017) ocasionan anomalías y más si se consumen ácidos saturados o trans que pueden propiciar riesgos a la salud del consumidor (Moghadasian y Shahidi, 2016).

Por ello, en los últimos tiempos las grasas poliinsaturadas (PUFA) o ácidos grasos volátiles son de gran interés puesto que se encuentran en cantidades versátiles en membranas celulares de especies mamíferas entre las más importantes están: el ácido docosahexaenoico (DHA) y el eicosapentaenoico (EPA), de la familia de omega-3, en donde los mariscos son la fuente más rica de estos componentes, mientras el ácido $\alpha$-linoleico se encuentra en vegetales como aceites de semillas, hojas verdes y leguminosas (Colussi et al., 2016). Estos compuestos propician una disminución en el número de personas que presentan enfermedades cardiovasculares y en aquellos que mueren por esa causa según estudios epidemiológicos de (Baker, Miles, Burdge y Yaqoob, 2016).

Rasti, Erfanian y Selemat (2017) han desarrollado una serie de evaluaciones acerca del comportamiento e influencia en la parte sensorial en productos de alto consumo como el pan y la leche con omega $(\omega 3)$ microencapsulado en forma de nanoliposomas, encontrando así un contenido de PUFAs entre (4,2-6,5 \% y 5,6-5,9\%) respectivamente, logrando una alta estabilidad durante el almacenamiento sin ningún sabor desagradable mencionado por los panelistas. También se han implementado estrategias con el fin de mejorar la composición de productos lácteos con relación a la cantidad de ácidos grasos presentes. Santurino, Calvoa, Gómez y Fontechaa (2017) han elaborado un queso de cabra natural enriquecido en ácido linoleico conjugado (CLA) y omega-3, a partir de un suplemento suministrado al animal de linaza 
extruida, en donde se obtuvo que el contenido de omega-3 fue 5 veces más alto que el control sin variaciones significativas en su textura y calidad sensorial durante el almacenamiento, reduciendo así el contenido de colesterol y beneficiado a personas con problemas de sobrepeso. Del mismo modo se ha sustituido mezclas de grasa comerciales por aceites vegetales o semillas oleaginosas, aceite de pescado o en combinación, agregando de esta forma un valor funcional al alimento.

Por otra parte, en la industria avícola se ha incrementado el interés de enriquecer la dieta de las aves con aceite de pescado, linaza o colza, debido al aumento de los ácidos grasos poliinsaturados que se generan en la carne. Estudios realizados han demostrado que los niveles de este tipo de ácidos incrementan, encontrando así mayores niveles de EPA y DHA de la pechuga y muslos, al igual que los niveles de ácido linolénico en donde una porción de $100 \mathrm{~g}$ de carne de pechuga o muslo proporcionan en promedio el $33 \%$ y el $15,5 \%$ respectivamente, de la ingesta diaria recomendada de EPA y DHA para los seres humanos (Konieczka, Czauderna y Smulikowska, 2017).

\section{Compuestos fenólicos}

Estos componentes constituyen al grupo de los micronutrientes presentes en el reino vegetal importantes en la dieta humana, como: fenoles, ácidos fenólicos, antocianinas, triterpenos, compuestos sulfurados, resveratrol, tioalilos, entre otros, siendo metabolitos secundarios de las plantas que poseen propiedades de gran interés. Por esta razón, han generado el interés los investigadores gracias a los beneficios a la salud, debido al poder antioxidante que poseen; con ello se busca quelar metales y captar así radicales libres causantes del envejecimiento cutáneo, además de la influencia sensorial que estos compuestos le confieren a los alimentos (López, Ortega y Lozada, 2015; Tian Y. et al., 2016).

Un estudio realizado por Rius A. et al. (2015), acerca de la caracterización de los compuestos fenólicos en fibras procesadas de la industria de jugo en particular frutas como: manzana, pera, melocotón, naranja, mandarina, limón y zanahoria como vegetal, identificó la concentración de alrededor de cuarenta compuestos fenólicos, tanto los ligados al epicarpio (cáscara) como a del mesocarpio (pulpa). Por otra parte, los compuestos fenólicos de las aceitunas han generado gran inquietud en los investigadores, gracias a sus aportes benéficos en la salud, trayendo consigo importancia en el consumo de aceite de oliva debido a su poder antioxidante natural, de tal forma que se estime como un ingrediente de alimentos funcionales (Deng J et al., 2017). Adicionalmente, Chhouka et al. (2017) mencionan que los extractos de epicarpio (cáscara) de ajo contienen este tipo de compuestos con actividades antioxidantes y antibacterianas, que pueden ser relevantes en el uso de la industria alimentaria y farmacéutica.

Beretta V. et al. (2017) han encontrado con base a una medición de absorbancia la concentración de compuestos fenólicos como quercetina, miricetina, kaempferol, rutina, catequina, galato de epicatequina y epigalocatequina galato, en cebollas amarillas, rojas y blancas. Incluso, en la industria cafetera se ha encontrado que residuos poseen compuestos fenólicos con propiedades antioxidantes en aplicaciones farmacéuticas, alimentarias y de salud (Mayanga et al., 2017). Así mismo Vieira, Barreira y Oliveira (2016) identificaron dos compuestos fenólicos como ácido gálico y alfa catequina con propiedades antioxidantes y otros nutrientes en la cerveza liofilizada. Además, el extracto de levadura indica que puede ser un alimento interesante o ingrediente nutracéutico. Por tanto, su recuperación puede ser beneficiosa en términos de sostenibilidad e impacto ambiental al ser este un residuo industrial. De igual manera, dentro de los compuestos fenólicos de gran interés se encuentran los contenidos en el romero que ha mostrado actividad antiproliferativa y citotóxica con relación al cáncer colorectal (Ochoa et al., 2017). 


\section{Fitoestrógenos}

Las isoflavonas pertenecen al grupo de los fitoestrógenos, son compuestos bioactivos que poseen similitud estructural con los estrógenos y el consumo de estos compuestos tienen efectos positivos sobre el estado de salud (González y Durán, 2014; Markovic et al., 2015). Las isoflavonas se encuentran naturalmente en los vegetales, la soya y sus derivados son la mayor fuente de estas, pero recientemente se ha mostrado interés por otras fuentes, como las lentejas, garbanzos, fríjoles, habas, guisantes, algunas semillas y frutas (Vila-Donat et al., 2015).

Los primeros estudios para los fitoestrógenos se enfocaron en evitar el riesgo de contraer cáncer de mama y la potencialidad que tienen las isoflavonas como anticancerígeno; se evidenció que la administración de productos derivados de soya incide en la disminución de tumores de cáncer de mama en la menopausia, próstata, hígado, pulmón y esófago (González y Durán, 2014). Actualmente se ha demostrado que la soya tiene gran incidencia en la prevención de osteoporosis, utilizado como antioxidante y en la salud cardiovascular gracias a las proteínas e isoflavonas que esta contiene para reducir el riesgo de estas enfermedades (Carmignani, Orcesi, Costa y Pinto, 2014; Mendoza, Roa y Ahumada, 2015). Por otro lado, estudios epidemiológicos asocian que consumir cebada reduce las probabilidades de adquirir enfermedades del corazón, cáncer de colon, presión y cálculos biliares, debido a los componentes bioactivos que esta posee (flavonoides y lignanos) y la prevención que ejercen en el desarrollo de enfermedades crónicas (Idehen, Tang y Sang, 2017).

Mattioli et al. (2016) demostraron cómo enriquecen los compuestos bioactivos (incremento de fitoestrógenos como las isoflavonas, cumestanos y lignanos) a los huevos de gallina con suplementos de alfalfa y brotes de lino, lo que resulta en la disminución de colesterol en los huevos, destacando que estos pueden ser considerados un alimento funcional gracias a la composición que poseen. Hoy, los fitoquímicos bioactivos (flavonoides, lignanos, vitaminas, esteroles, entre otros) de la cebada, del trigo y el centeno cumplen un papel importante en prevención de ciertas enfermedades metabólicas, de alta presión de la sangre, del colon y crónicas (Andersson, Dimberg, Åman y Landberg, 2014; Idehen, Tang y Sang, 2017).

\section{Flavonoides}

En la industria agroalimentaria se emplean antioxidantes sintéticos para inhibir la oxidación de lípidos, de los cuales se encuentran principalmente el BHA (butil-hidroxil-anisol), BHT (butil-hidroxil-tolueno), TBHQ (terc-butilhidroquinona) y PG (propil galato), que han sido las causas de enfermedades cardiacas y agentes carcinogénicos (Kunrath et al., 2017). Aunque, con la intención de preservar la salud del consumidor, se ha invertido en la búsqueda de compuestos naturales con propiedades antioxidantes para sustituir a los sintéticos, en los que existen diversas alternativas de fuentes de alimentos, entre los cuales se encuentran las frutas, pero principalmente su cáscara, propóleo, cacao, entre otros (Martini et al., 2017).

Cada alimento posee una actividad biológica específica dentro del organismo humano, y esto depende normalmente de su composición nutricional. Compuestos como los flavonoides tienen efectos beneficiosos contra la salud humana, como el caso de la moringa que es un vegetal importante en los países en desarrollo por su diversa gama de nutrientes que pueden combatir enfermedades como obesidad, diabetes y cáncer, ya que contienen flavonoides como: quercitina y kaempferol, en hojas, raíz, flor y el recubrimiento de la semilla (Lin, Zhang y Chen, 2018).

En el caso de alimentos ricos en compuestos polifenólicos, como miel de abejas y propóleo, esta actividad especialmente la hacen los flavonoides, que son los responsables de brindar propiedades antiinflamatorias, antimicrobianas y antivirales, y también la característica antioxidante (Biluca et al., 2016; Machado et al., 2016; Raffa et al., 2017; Rao, Krishnan, Salleh y Gan, 2016). Los 
resultados de estudios aleatorios sugieren que las antocianidinas de bayas y flavanoides-3-ols de té verde y cacao pueden disminuir la existencia de enfermedades cardiovasculares y el peligro de diabetes tipo 2 (Abd Eldaim, Abd Elrasoul y Abd Elaziz, 2017), por otro lado, extractos obtenidos de hojas de Ginkgo biloba con alto contenido de flavonoides es utilizado por sus propiedades antioxidantes y como tratamiento de Alzheimer, debido a propiedades estabilizadoras de membrana neuronal (Rivadeneyra-Domínguez et al., 2017).

En la actualidad se conocen más de 8000 compuestos fenólicos que se clasifican como flavonoides, siendo su principal fuente de alimentos de característica funcional las frutas, verduras, semillas y flores; dentro de los alimentos consumidos con frecuencia están el té, vino, cerveza y otras bebidas de la misma derivación (Navarro, Periago y Garcìa, 2017). Estos compuestos ayudan a inhibir de forma natural la inflamación de la mucosa intestinal (Hoensch y Oertel, 2015). Según Porto et al. (2016), la exposición a la luz induce la producción de flavonoides en las frutas que, junto con los carotenoides, son pigmentos vegetales que protegen al organismo de los rayos UV; se encuentran, por ejemplo, en la cáscara del tomate. Kunrath et al, (2017) comprobaron una de las funciones principales de los flavonoides, al realizar un recubrimiento de propóleo a bajas concentraciones a un salami, y evaluar su acción antioxidante al inhibir la oxidación de lípidos presentes en este alimento. Otra investigación enfocada a los flavonoides por su acción antioxidante la hace Tenorio, (2016), quien obtiene del epicarpio de naranja (Citrus paradisi x Citrus reticulata) extractos a diferentes concentraciones de flavonoides y posteriormente lo aplica al aceite vegetal sacha inchi; así, concluye que a mayor concentración de flavonoides en el aceite, existe menor índice de peróxidos y el tiempo de vida útil aumenta (Tabaldi et al., 2016; Nunez et al., 2016; Morales et al., 2016).

\section{Carotenoides}

Los compuestos antioxidantes, normalmente reportados en alimentos funcionales, son capaces de inhibir o retrasar las lesiones causadas por los radicales libres, que son moléculas con uno o más electrones no emparejados que reaccionan rápidamente con diferentes blancos celulares causando daños que están asociados con enfermedades degenerativas y envejecimiento (Vizzoto et al., 2017). Las reacciones de los radicales libres son compensadas por la acción de antioxidantes obtenidos a través de la dieta, como ácido ascórbico, $\alpha$-tocoferol, carotenoides y polifenoles.

Los carotenoides se encuentran en la mayoría de estructuras vegetales y son los responsables de la coloración roja, amarilla y naranja. También son uno de los grupos más importantes de pigmentos naturales debido a su amplia distribución, diversidad estructural y muchas funciones medicinales por su acción antioxidante (Lourenzi et al., 2016; Gómez et al., 2017). Las patatas dulces tienen altos niveles de compuestos bioactivos como antocianinas y $\beta$-caroteno que se describen con propiedades antioxidantes y antimutágenas. La principal característica de los carotenoides presentes en las patatas es su actividad de provitamina A (Vizzoto et al., 2017; Oliveira et al., 2017). Por otra parte, Quintero et al. (2014) realizaron una sustitución con harina de alga (Ulva clathrata) rica en carotenoides en la tortilla de maíz comúnmente usada en México, con resultados positivos como agente antioxidante y además incrementó las concentraciones de fibra, proteína y minerales.

En la tabla 1 se presenta un resumen y algunos ejemplos de estudios realizados en los últimos cuatro años, sobre la aplicación de los diferentes compuestos bioactivos nombrados anteriormente con el fin de obtener alimentos funcionales. 
Tabla 1. Ejemplos de estudios sobre compuestos bioactivos en alimentos funcionales con beneficios potenciales en la salud humana

\begin{tabular}{|c|c|c|c|}
\hline $\begin{array}{c}\text { Compuestos } \\
\text { bioactivos }\end{array}$ & $\begin{array}{c}\text { Ejemplos potenciales de aplicación en } \\
\text { agroindustria }\end{array}$ & Beneficios potenciales & Referencias \\
\hline $\begin{array}{l}\text { Probióticos, pre- } \\
\text { bióticos y simbió- } \\
\text { ticos }\end{array}$ & $\begin{array}{l}\text { Encapsulación por liofilización de ce- } \\
\text { pas probióticas en mezclas con agentes } \\
\text { prebióticos durante el almacenamiento de } \\
\text { alimentos, dietas de ganado con productos } \\
\text { simbióticos. }\end{array}$ & $\begin{array}{l}\text { Regula el tránsito intestinal } \\
\text { y equilibra la flora intestinal, } \\
\text { reduce problemas asociados } \\
\text { a cáncer de colon. }\end{array}$ & $\begin{array}{l}\text { Rodríguez et al. (2015); } \\
\text { Salazar et al. (2015); Olveira } \\
\text { y González (2016). }\end{array}$ \\
\hline Fibra dietaria & $\begin{array}{l}\text { Adición de fibras vegetales en productos } \\
\text { de panificación, producción de galletas, } \\
\text { helados, alimentos cárnicos y concentrados } \\
\text { a partir de fibra obtenida de epicarpio de } \\
\text { vegetales. }\end{array}$ & $\begin{array}{l}\text { Reducción del estreñimiento } \\
\text { y buen funcionamiento del } \\
\text { colon, reducción del coleste- } \\
\text { rol y niveles de glucosa en la } \\
\text { sangre. }\end{array}$ & $\begin{array}{l}\text { Alarcón et al. (2015); Crizel } \\
\text { (2015); Guedes et al. (2016); } \\
\text { Torres et al. (2016); Okiyama } \\
\text { et al. (2017); Benítez et al. } \\
\text { (2018); Djurle et al. (2018). }\end{array}$ \\
\hline Ácidos grasos & $\begin{array}{l}\text { Enriquecimiento con omega } 3 \text { y } 6 \text { en hue- } \\
\text { vo, panificación, productos cárnicos como } \\
\text { el pollo (con ayuda del suplemento de } \\
\text { compuestos como linaza en la alimentación } \\
\text { de los animales), aceite en semillas, hojas } \\
\text { de leguminosas y pescado. }\end{array}$ & $\begin{array}{l}\text { Ejercen protección a nivel } \\
\text { cardiovascular como hiper- } \\
\text { tensión o isquemia como } \\
\text { agente antiinflamatorio. }\end{array}$ & $\begin{array}{l}\text { Colussi et al. (2016); Rasti } \\
\text { B. et al. (2017); Konieczka, } \\
\text { Czauderna y Smulikowska, } \\
\text { (2017). }\end{array}$ \\
\hline $\begin{array}{l}\text { Compuestos fenó- } \\
\text { licos }\end{array}$ & $\begin{array}{l}\text { Enriquecimiento de compuestos fenólicos } \\
\text { en jugos de fruta como: manzana, pera, } \\
\text { melocotón, naranja, mandarina, limón y } \\
\text { zanahoria como vegetal, alto contenido en } \\
\text { aceite de oliva, extractos de cáscara de ajo, } \\
\text { cebollas, al igual que en el romero, morin- } \\
\text { ga y en cerveza liofilizada. }\end{array}$ & $\begin{array}{l}\text { Presentan un efecto an- } \\
\text { tioxidante y ejercen efectos } \\
\text { favorables sobre el perfil } \\
\text { lipídico al igual que acción } \\
\text { antimicrobiana. }\end{array}$ & $\begin{array}{l}\text { Rius et al. (2015); Vieira } \\
\text { et al. (2016); Deng et al. } \\
\text { (2017); Chhouka et al. } \\
\text { (2017); Beretta et al., (2017). }\end{array}$ \\
\hline Fitoestrógenos & $\begin{array}{l}\text { Enriquecimiento de fitoestrógenos en hue- } \\
\text { vos de gallina, alimentos derivados de soya, } \\
\text { trigo, centeno. }\end{array}$ & $\begin{array}{l}\text { Previene osteoporosis, anti- } \\
\text { oxidantes, anticancerígenos, } \\
\text { reducción riesgo enfermeda- } \\
\text { des crónicas. }\end{array}$ & $\begin{array}{l}\text { Mattioli et al. (2016); Idehen } \\
\text { et al. (2017); Raheja, Gird- } \\
\text { har, Lather y Pandita (2018). }\end{array}$ \\
\hline Flavonoides & $\begin{array}{l}\text { Recubrimiento con propóleo en carnes para } \\
\text { evaluar la acción antioxidante. Aplicación } \\
\text { de extractos de hojas y flores comestibles. }\end{array}$ & $\begin{array}{l}\text { Presentan una eficacia en el } \\
\text { control del proceso oxidati- } \\
\text { vo de los lípidos, alargando } \\
\text { el tiempo de vida útil en } \\
\text { productos. }\end{array}$ & $\begin{array}{l}\text { Biluca et al. (2016); Macha- } \\
\text { do et al. (2016); Kunrath et } \\
\text { al. (2017). }\end{array}$ \\
\hline Carotenoides & $\begin{array}{l}\text { Enriquecimiento de harina en tortillas, mi- } \\
\text { croencapsulación de licopeno en pasteles, } \\
\text { curmuma en helado y yogur, carotenoides } \\
\text { en ácidos grasos, provitamina A, enriqueci- } \\
\text { miento de snack, aprovechamiento de sub- } \\
\text { productos agroindustriales como colorantes } \\
\text { y antioxidantes. }\end{array}$ & $\begin{array}{l}\text { Son una importante fuente } \\
\text { de carotenoides que tienen } \\
\text { propiedades antioxidantes, } \\
\text { además proporcionan benefi- } \\
\text { cios en la función cognitiva, } \\
\text { ocular y cardiovascular. }\end{array}$ & $\begin{array}{l}\text { Rutz et al. (2016); Martí- } \\
\text { nez-Girón, Figueroa-Molano } \\
\text { y Ordóñez-Santos (2017); } \\
\text { Eggersdorfer y Wiss (2018). }\end{array}$ \\
\hline
\end{tabular}

Fuente: elaboración propia.

\section{CONCLUSIONES}

Este trabajo de revisión tuvo como objetivo presentar una recuperación de fuentes bibliográficas en relación con la importancia de los compuestos bioactivos presentes en alimentos funcionales y sus diferentes aplicaciones en la agroindustria. Los resultados indicaron que cada día son más los investigadores que centran su interés en el estudio de alimentos funcionales, debido a que el consumo de alimentos que aporten al nivel nutricional y mitiguen acciones no benéficas que se producen 
en los seres humanos por el consumo de alimentos poco saludables, generan un efecto crucial en la salud y originan un gran interés para el campo científico. Por este motivo, los alimentos denominados funcionales, han Ilamado la atención de la población mundial, debido a que presentan compuestos bioactivos como: prebióticos, probióticos, simbióticos, fibras (solubles e insolubles), ácidos grasos como el omega 3 y 6, compuestos fenólicos, fitoestrógenos, flavonoides y carotenoides; cumpliendo un papel funcional en el organismo. A partir de lo anterior surge la necesidad de enriquecer o fortificar los alimentos con estos componentes y potencializar su actividad, debido a que muchos de estos no se producen en el metabolismo humano.

\section{REFERENCIAS}

Abd Eldaim, M.A., Abd Elrasoul, A.S. y Abd Elaziz, S.A. (2017). An aqueous extract from Moringa oleifera leaves ameliorates hepatotoxicity in alloxan-induced diabetic rats. Biochemistry and Cell Biology, 95, 524-530. DOI: https://doi.org/10.1139/ bcb-2016-0256

Alarcón, M., López, J. y Restrepo, D. (2015). Frutas co-productos agroindustriales en Colombia, sus fuentes y usos potenciales en las industrias de alimentos procesados: una revisión. Revista Facultad Nacional de Agronomía, 68(2), 7729-7742.

Andersson, A., Dimberg, L., Åman, P. y Landberg, R. (2014). Recent findings on certain bioactive components in whole grain wheat and rye. Journal of Cereal Science, 59(3), 294-311. DOI: https://doi.org/10.1016/j.jcs.2014.01.003

Azevedo, R., Fosse, J., Pereira, S., Cardoso, L., Andrade, D. y Vidal, J. (2016). Dietary mannan oligosaccharide and Bacillus subtilis in diets for Nile tilapia (Oreochromis niloticus). Acta Scientiarum. Animal Sciences, 38(4), 347-353. DOI: https://doi. org/10.4025/actascianimsci.v38i4.31360

Baker, J., Miles, A., Burdge, C., Yaqoob, C. (2016). Metabolism and functional effects of plant-derived omega-3 fatty acids in humans. Progress in
Lipid Research, 64, 30-56. DOI: https://doi.org/10.1016/j.plipres.2016.07.002

Barazarte, H., Sangronis, E., Moreno, I., Garmendia, C. y Mujica, Y. (2015). Laminados de guayaba (Psidium guajava $L$.) enriquecidos con inulina y calcio. Archivos Latinoamericanos de Nutrición, 65(4), 225-233.

Benítez, V., Esteban, R., Moniz, E., Casado, N., Aguilera, Y. y Mollá, E. (2018). Breads fortified with wholegrain cereals and seeds as source of antioxidant dietary fiber and other bioactive compounds. Journal of Cereal Science, 82, 113-120. DOI: https:// doi.org/10.1016/j.jcs.2018.06.001

Biluca, F. C., Braghini, F., Gonzaga, L.V., Oliveira, A.C. y Fett, R. (2016). Physicochemical profiles, minerals and bioactive compounds of stingless bee honey (Meliponinae). Journal of Food Composition and Analysis, 50, 61-69. DOI: https://doi.org/10.1016/j. jfca.2016.05.007

Beretta, V., Bannoud, F., Insani. M., Galmarini, C. y Cavagnaro, P. (2017). Dataset on absorption spectra and bulb concentration of phenolic compounds that may interfere with onion pyruvate determinations. Data in Brief, 11, 208-213. DOI: https://doi. org/10.1016/j.dib.2017.01.015

Blanco, M.S., León, A.E. y Ribotta, P.D. (2018). Incorporation of dietary fiber on the cookie dough. Effects on thermal properties and water availability. Food Chemistry, 271, 309-317. DOI: https://doi.org/10.1016/j.foodchem.2018.07.146

Boer, A., Urlings, M., Bast, A. (2016). Active ingredients leading in health claims on functional foods. Journal of Functional Foods, 20, 587-593. DOI: https:// doi.org/10.1016/j.jff.2015.11.025

Cadena, R., Caimi, D., Jaunarena, I., Lorenzo, I., Vidal, L., Ares, G., Deliza, R. y Giménez, A. (2014). Comparison of rapid sensory characterization methodologies for the development of functional yogurts. Food Research International, 64, 446-455. DOI: https://doi.org/10.1016/j.foodres.2014.07.027

Carmignani, L., Orcesi, A., Costa, L. y Pinto, A. (2014). The effect of soy dietary supplement and low dose of hormone therapy on main cardiovascular health biomarkers: a randomized controlled 
trial. Revista Brasileira de Ginecologia e Obstetrícia, 36(6), 251-258. DOI: https://doi.org/10.1590/ S0100-720320140004976

Chasquibol, N., Lengua, L., Delmas, I., Rivera, D., Bazan, D., Aguirre, R., Bravo, M. (2014). Alimentos funcionales o fitoquímicos, clasificación e importancia. Revista Peruana de Química e Ingeniería Química, 6(2), 9-20.

Chhouka, K., Uemoria, C., Wahyudionoa., Kandaa, H. y Goto, M. (2017). Extraction of phenolic compounds and antioxidant activity from garlic husk using carbon dioxide expanded ethanol. Chemical Engineering \& Processing: Process Intensification. Nutrition, Metabolism \& Cardiovascular Diseases, 27, 191-200. DOI: https://doi.org/10.1016/j. cep.2017.03.023

Cigarran, S., González, E. y Cases, A. (2017). Microbiota intestinal en la enfermedad renal crónica, Nefrología, 37(1), 9-19. DOI: https://doi.org/10.1016/j.nefro.2016.05.008

Colussi, G., Catena, C., Novello, M., Bertin, N. y Sechi. (2016). Impact of omega-3 polyunsaturated fatty acids on vascular function and blood pressure: Relevance for cardiovascular outcomes. Nutrition, Metabolism \& Cardiovascular Diseases, 27, 191-200. DOI: https://doi.org/10.1016/j. numecd.2016.07.011

Crizel, T., Ríos, A., Thys, R., Hickmann, S. (2015). Efectos de la incorporación de fibra de naranja subproducto en las propiedades funcionales y tecnológicas de la pasta. Ciencia y Tecnología de Alimentos (Campinas), 35(3), 546-551. DOI: https:// doi.org/10.1590/1678-457X.6719

Deng, J., Xu, Z., Xiang, C., Liu, J., Zhou, L., Li, T., Yang, Z. y Ding, C. (2017). Comparative evaluation of maceration and ultrasonic-assisted extraction of phenolic compounds from fresh olives. Ultrasonics Sonochemistry, 37, 328-334. DOI: https://doi.org/10.1016/j.ultsonch.2017.01.023

Djurle, S., Andersson, A. y Andersson, R. (2018). Effects of baking on dietary fibre, with emphasis on $\beta$-glucan and resistant starch, in barley breads. Journal of Cereal Science, 79, 449-455. DOI: https:// doi.org/10.1016/j.jcs.2017.10.017
Eggersdorfer, M. y Wyss, A. (2018). Carotenoids in human nutrition and health. Archives of Biochemistry and Biophysics, 652, 18-26. DOI: https://doi. org/10.1016/j.abb.2018.06.001

Fuentes, L., Acevedo, D. y Gelvez, V. (2015). Alimentos funcionales: impacto y retos para el desarrollo y bienestar de la sociedad colombiana. Biotecnología en el Sector Agropecuario y Agroindustrial, 13(2)- 140-149. DOI: https://doi.org/10.18684/ BSAA(13)140-149

Gómez, J., Correa, A., Coutinho, K., Paiva, M., Fernandes, A., Rodriguez, V. y Stringheta, P. (2017). Beverages formulated with whey protein and added lutein. Ciencia Rural, 47(3), 1-7.

González, N. y Durán, S. (2014). Isoflavonas de soya y evidencias sobre la protección cardiovascular. Nutrición Hospitalaria, 29(6), 1271-1282.

Guedes, J., M., Salgado, R., Costa, B., Guedes, J. y Conte, C. (2016). Washed cashew apple fiber (Anacardium occidentale L.) as fat replacer in chicken patties. LWT-Food Science and Technology, 71, 268-273. DOI: https://doi.org/10.1016/j.Iwt.2016.04.005

Hevia, A., Delgado, S., Sánchez, B. y Margolles, A. (2015). Molecularplayers involved in the interaction between beneficialbacteria and the immune system. Frontier in Microbiology, 6, 1285-1290. DOI: https://doi.org/10.3389/fmicb.2015.01285

Hoensch, H. y Oertel, R. (2015). The value of flavonoids for the human nutrition: Short review and perspectives. Clinical Nutrition Experimental, 3, 8-14. DOI: https://doi.org/10.1016/j.yclnex.2015.09.001

Idehen, E., Tang, Y. y Sang, S. (2017). Bioactive phytochemicals in barley. Journal of Food and Drug Analysis, 25(1), 148-161. DOI: https://doi.org/10.1016/j. jfda.2016.08.002

Illanes, A. (2015). Alimentos funcionales y biotecnología. Revista Colombiana de Biotecnología, 17(1), 5-8. DOI: https://doi.org/10.15446/rev.colomb.biote.v17n1.50997

Konieczka, P., Czauderna, M., Smulikowska, S. (2017). The enrichment of chicken meat with omega-3 fatty acids by dietary fish oil or its mixture with rapeseed or flaxseed: Effect of feeding duration Dietary fish oil, flaxseed, and rapeseed and n-3 enriched 
broiler meat. Animal Feed Science and Technology, 223, 42-52. DOI: https://doi.org/10.1016/j. anifeedsci.2016.10.023

Kunrath, C., Savoldl, D., Mieski, J., Novello, C., Trindade, A., Marchi, J. y Benedetti, I. (2017). Application and evaluation of propolis, the natural antioxidant in Italian-type salami. Brazilian Journal of Food Technology, 20, 1-10. DOI: https://doi. org/10.1590/1981-6723.3516

Lemahieu, C.; Bruneel, C.; Muylaert, K.; Buyse, J. y Foubert, I. (2017). Microalgal Feed Supplementation to Enrich Eggs with Omega-3 Fatty Acids. Egg Innovations and Strategies for Improvements. 36, 383-391. DOI: https://doi.org/10.1016/ B978-0-12-800879-9.00036-6

Lin M., Zhang J. y Chen X. (2018). Bioactive flavonoids in Moringa oleifera and their health- promoting properties. Journal of Functional Foods, 47, 469-479. DOI: https://doi.org/10.1016/j.jff.2018.06.011

López, L., Ortega, A. y Lozada, J. (2015). Modificaciones enzimáticas de compuestos fenólicos. Temas Selectos de Ingeniería de Alimentos, 9(1), 5-14.

Lourenzi, C., Clemente, E., Menezes, D., Martins, K. y Correia, J. (2016). Effects of 1-MCP onthe post-harvest quality of the orange $\mathrm{cv}$. Pera stored under refrigeration. Ciência Agronômica, 47, 624-632.

Machado, A., Mostarda, C., Irigoyen, M. y Rigatto, K. (2016). A single dose of dark chocolate increases parasympathetic modulation and heart rate variability in healthy subjects. Nutrición Campinas, 29, 765-773.

Markovic, R., Baltic, M., Pavlovic, M., Glisic, M., Radulovic, S., Djordjevic, V. y Sefer, D. (2015). Isoflavones-from Biotechnology to Functional Foods. Procedia Food Science, 5, 176-179. DOI: https:// doi.org/10.1016/j.profoo.2015.09.050

Martini, D., Feliciane, G., Matias, R., Camargo, W. y Trombini, N. (2017). Seasonality on the antifungal potential of green propolis collected in Campo Grande-MS, Brazil. Ciencia Rural, 47(3), 1-6. DOI: https://doi.org/10.1590/0103-8478cr20160312

Martínez-Girón, J., Figueroa-Molano, A.M. y Ordóñez-Santos, L.E. (2017). Effect of the addition of peach palm (Bactris gasipaes) peel flour on the color and sensory properties of cakes. Food Science and Technology, 37(3), 418-424. DOI: https:// doi.org/10.1590/1678-457x.14916

Mattioli, S., Dal Bosco, A., Martino, M., Ruggeri, S., Marconi, O., Sileoni, V., Falcinelli, B., Castellini, C. y Benincasa, P. (2016). Alfalfa and flax sprouts supplementation enriches the content of bioactive compounds and lowers the cholesterol in hen egg. Journal of Functional Foods, 22, 454-462. DOI: https://doi.org/10.1016/j.jff.2016.02.007

Mayanga, P., Lachaos, D., Rezende, C., Prado, J., Ma, Z., Tompsett, G., Timko, M. y Forster, T. (2017). Valorization of coffee industry residues by subcritical water hydrolysis: Recovery of sugars and phenolic compounds. Journal of Supercritical Fluids, 120, 75-85. DOI: https://doi.org/10.1016/j. supflu.2016.10.015

Mendoza, D., Roa, C. y Ahumada, C. (2015). Efecto de las isoflavonas de la soja en la salud ósea de adultos y niños. Revista Salud Uninorte, 31(1), 138-152.

Moghadasian, M. y Shahidi, F. (2016). Fatty Acids. Module in Biomedical Sciences. International Encyclopedia of Public Healt, 2, 114-112.

Morales, R., Lima, W., Guedes, J., Branco, A. y Matiazu, M. (2016). Antibacterial potential of native plants from the caatinga biome against Staphylococcus spp. Isolates from small ruminants with mastitis. Revista Caatinga, 29, 758-763. DOI: https://doi. org/10.1590/1983-21252016v29n328rc

Narbona, E., Uberos, J., Armadá, M., Couce, M.L., Rodríguez, G. y Saenz, M. (2014). Grupo de Nutrición y Metabolismo Neonatal, Sociedad Española de Neonatología: recomendaciones y evidencias para la suplementación dietética con probióticos en recién nacidos de muy bajo peso al nacer. Anales de Pediatría, 81(6), 397-408.

Navarro, I., Periago, M. y Garcìa, F. (2017). Estimación de la ingesta diaria de compuestos fenólicos en la población española. Revista Española de Nutrición Humana y Dietética, 21, 320-326. DOI: https://doi. org/10.14306/renhyd.21.4.357

Nunez, R., Cordero, M., Cabral, F., Marques, T., Quilty, B., Da Silva, R. y McGuinness, G. (2016). FTIR analysis and quantification of phenols and flavonoids of 
five commercially available plants extracts used in wound healing. Matéria, 21, 767-779. DOI: https:// doi.org/10.1590/S1517-707620160003.0072

Ochoa, A., Linares, I., Pérez, A., Barrajón, E., González, I., Arraez, D., Micol, V. y Segura A. (2017). Phenolic compounds in rosemary as potential source of bioactive compounds against colorectal cancer: In situ absorption and metabolism study. Journal of Functional Foods, 33, 202-210. DOI: https://doi.org/10.1016/j.jff.2017.03.046

Okiyama, D., Navarro, S. y Rodrigues, C. (2017). Cocoa shell and its compounds: Applications in the food industry. Trends in Food Science \& Technology, 63, 103-112. DOI: https://doi.org/10.1016/j. tifs.2017.03.007

Olveira, G. y González-Molero, I. (2016). An update on probiotics, prebiotics and symbiotics in clinical nutrition. Endocrinología y Nutrición, 63(9), 482-494. DOI: https://doi.org/10.1016/j. endonu.2016.07.006

Oliveira, L., Macedo, S., Oliveira, A., Pacheco, S., Pereira, S. y Martins, V. (2017). Avaliação do armazenamento a frio sobre os compostos bioativos e as características físico-químicas e microbiológicas do suco de umbu pasteurizado. Brazilian Journal of Food Technology, 20, 1-8.

Porto J., Hojo, T., Batista, M., Pereira, N., Lacerda, O. y Quieroz, J. (2016). Quality and antioxidant activity of totamto cultivated under different sources and doses of nitrogen. Revista Caatinga, 29,780-788. DOI: https://doi.org/10.1590/1983-21252016v29n401 rc

Quintero, A., Gonzalez, G., Solano, A., Reyes, G., Villanueva, J. y Bravo, G. (2014). Caracterización de una tortilla tostada elaborada con maíz (Zea mays) y alga (Ulva clathrata) como prospecto de alimento funcional. Journal of Community Nutrition, 20, 22-28.

Raheja, S., Girdhar, A., Lather, V. y Pandita, D. (2018). Biochanin A: A phytoestrogen with therapeutic potential. Trends in Food Science \& Technology, 79, 55- 66. DOI: https://doi.org/10.1016/j. tifs.2018.07.001

Raffa, D., Maggio, B., Raimondi, V., Plescia, F. y Daidone, G. (2017). Recent discoveries of anticancer flavonoids. European Journal of Medicinal Chemestry, 142, 213-228. DOI: https://doi.org/10.1016/j. ejmech.2017.07.034

Rana, S., Gupta, S., Rana, A. y Bhushan, S. (2015). Functional properties, phenolic constituents and antioxidant potential of industrial apple pomace for utilization as active food ingredient. Food Science and Human Wellness, 4(4), 180-187. DOI: https:// doi.org/10.1016/j.fshw.2015.10.001

Rao, P., Krishnan, K., Salleh, N. y Gan, S. (2016). Biological and therapeutic effects of honey produced by honey bees and stingless bees: a comparative review. Revista Brasileira de Farmacognosia, 26(5), 657-664. DOI: https://doi.org/10.1016/j. bjp.2016.01.012

Rasti, B., Erfanian, A. y Selamat, J. (2017). Novel nanoliposomal encapsulated omega-3 fatty acids and their applications in food. Food Chemistry, 230, 690-696. DOI: https://doi.org/10.1016/j. foodchem.2017.03.089

Reis, J., Pinheiro, M., Oti, A., Feitosa, D., Pantoja, M. y Barros, R. (2016). Technological information regarding prebiotics and probiotics nutrition versus the patent registers: what is new? Abcd. Arquivos Brasileiros de Cirurgia Digestiva (São Paulo), 29(4), 279-281. DOI: https://doi. org/10.1590/0102-6720201600040016

Ribeiro, F., Mendes, J., Francisco, C., Castilhos, A., Pariz, C. y Silva, M. (2015). Simbióticos e monensina sódica no desempenho e na qualidade da carne de novilhas mestiças Angus confinadas. Pesquisa Agropecuária Brasileira, 50(10), 958-966. DOI: https://doi.org/10.1590/S0100-204X2015001000012

Rius, A., Eras, J., Vilaro, F., Cubero, M., Balcells, M. y Garayoa, R. (2015). Characterization of phenolic compounds in processed fibers from the juice industry. Food Chemistry, 172, 575-584. DOl: https://doi.org/10.1016/j.foodchem.2014.09.071

Rivadeneyra-Domínguez, E., Vázquez-Luna, A., Rodríguez-Landa, J.F., Mérida-Portilla, C.V. y Díaz-Sobac, R. (2017). Efecto protector de 2 presentaciones comerciales de Ginkgo biloba sobre las alteraciones motoras inducidas por el jugo de yuca (Manihot esculenta Crantz) en la rata Wistar. Neurología, 
32(8), 516-522. DOI: https://doi.org/10.1016/j. nrl.2016.02.018

Rodríguez, S., Giraldo, G. y Zuluaga, Y. (2015). Evaluación de la incorporación de fibra prebiótica sobre la viabilidad de LactobaciHus casei impregnado en matrices de mora (Rubus glaucus). Información Tecnológica, 26(5), 25-34. DOI: https://doi. org/10.4067/S0718-07642015000500005

Rodríguez, S., Giraldo, G. y Montes, L. (2016). Encapsulación de alimentos probióticos mediante liofilización en presencia de prebióticos. Información tecnológica, 27(6), 135-144. DOI: https://doi. org/10.4067/S0718-07642016000600014

Rutz, J., Borges, C., Zambiazi, R., da Rosa, C. y da Silva, M. (2016). Elaboration of microparticles of carotenoids from natural and synthetic sources for applications in food. Food Chemestry, 202. 324-333. DOI: https://doi.org/10.1016/j.foodchem.2016.01.140

Salas, G., Salcedo, O. y Aguilera, M. (2013). Análisis de indicadores socioeconómicos y su impacto en la salud pública de Bogotá. Revista Tecnura, 17, 134-148.

Salazar, B., Cortés, M. y Montoya, O. (2015). El impacto de las condiciones de almacenamiento sobre la estabilidad de la caña de azúcar en polvo biofortificados con granos de kéfir. Revista Facultad Nacional de Agronomía, 68(2), 7703-7712.

Santurino, C., Calvoa, M.V., Gómez, C. y Fontechaa, J. (2017). Characterization of naturally goat cheese enriched in conjugated linoleic acid and omega-3 fatty acids for human clinical trial in overweight and obese subjects. Pharma Nutrition, 5(1), 8-17. DOI: https://doi.org/10.1016/j.phanu.2016.12.001

Serna, L. y Torres, C. (2015). Potencial agroindustrial de cáscaras de mango (Mangifera indica) variedades Keitt y Tommy Atkins. Acta Agronómica, 64(2), 110-115. DOI: https://doi.org/10.15446/acag. v64n2.43579

Suárez C., J.L., Restrepo M., J.W., Quinchía F., A. y Mercado N., F.A. (2017). Fibras vegetales colombianas como refuerzo en compuestos de matriz polimérica. Revista Tecnura, 21(51), 57-66. DOI: https://doi. org/10.14483/udistrital.jour.tecnura.2017.1.a04
Tabaldi, L., Vieira, M., Heredia, N., Nazari, A., Pilecco, M., Silva, L., Pereira, K., Cauz, L. y Lima C. (2016). Biomass yield and flavonoid and phenol content of Schinus terebinthifolius cultivated in single or double row with poultry litter. Ciencia Florestal, 26, 787796. DOI: https://doi.org/10.5902/1980509824207

Tenorio, M. (2016). Flavonoides extraídos de la cáscara de naranja tangelo (Citrus reticulada x Citrus paradisi) y su aplicación como antioxidante natural en el aceite vegetal sacha inchi (Plukenetia volubilis). Sciencia Agropecuaria, 7, 419-431. DOI: https:// doi.org/10.17268/sci.agropecu.2016.04.07

Tian, Y., Liimatainen, J., Alanne, A., Lindstedt, A., Liu, P., Sinkkonen, J., Kallio, H. y Yang, B. (2017). Phenolic compounds extracted by acidic aqueous ethanol from berries and leaves of different berry plants. Food Chemistry, 220, 266-281. DOI: https://doi. org/10.1016/j.foodchem.2016.09.145

Torres, J., González, K., Acevedo, D. y Jaimes, J. (2016). Efecto de la utilización de harina de Lens culinaris como extensor en las características físicas y aceptabilidad de una salchicha. Revista Tecnura, 20(48), 15-28. DOI: https://doi.org/10.14483/udistrital. jour.tecnura.2016.3.a01

Trescastro, E. y Bernabeu, J. (2015). Alimentos funcionales: ¿necesidad o lujo? Revista Española de Nutrición Humana y Dietética, 19(1), 1-3. DOI: https:// doi.org/10.14306/renhyd.19.1.153

Tur, J.A. y Bibiloni, M.M. (2015). Functional Foods. Encyclopedia of Food and Health, 1, 157-161.

Vieira, B., Barreira, M. y Oliveira, B. (2016). Natural phytochemicals and probiotics as bioactive ingredients for functional foods: Extraction, biochemistry and protected-delivery technologies. Trends in Food Science \& Technology, 50, 144-158. DOI: https://doi.org/10.1016/j.tifs.2015.12.007

Vila-Donat, P., Caprioli, G., Maggi, F., Ricciutelli, M., Torregiani, E., Vittori, S. y Sagratini, G. (2015). Effective clean-up and ultra-high-performance liquid chromatography-tandem mass spectrometry for isoflavone determination in legumes. Food Chemistry, 174, 487-494. DOI: https://doi.org/10.1016/j. foodchem.2014.11.047 
Vizotto, M., Dos Santos, E., Rocha, J., Cardoso, P., Lettnin, N., Suita, L. y Richter, A. (2017). Physicochemical and antioxidant capacity analysis of colored sweet potato genotypes: in natura and thermally processed. Ciencia Rural, 47(4), 1-8. DOI: https:// doi.org/10.1590/0103-8478cr20151385
Yan, L., Xiong, C., Qu, H., Liu, C., Chen, W. y Zheng, L. (2017). Non-destructive determination and visualization of insoluble and soluble dietary fiber contents in fresh-cut celeries during storage periods using hyperspectral imaging technique. Food Chemistry, 228, 249-256. DOI: https://doi. org/10.1016/j.foodchem.2017.02.010

\section{(c) (i) (2)

